\title{
Liposuction in the Treatment of Lipedema: A Longitudinal Study
}

\author{
Mehran Dadras $^{1,2}$, Peter Joachim Mallinger ${ }^{3}$, Cord Christian Corterier ${ }^{1}$, Sotiria Theodosiadi ${ }^{1}$, \\ Mojtaba Ghods ${ }^{1}$ \\ ${ }^{1}$ Department of Plastic Surgery, Klinikum Ernst von Bergmann, Potsdam; ${ }^{2}$ Department of Plastic Surgery, BG University Hospital \\ Bergmannsheil, Ruhr-University Bochum, Bochum, Germany; ${ }^{3}$ Department of Plastic Surgery, Klinikum Klagenfurt am Wörthersee, \\ Klagenfurt am Wörthersee, Austria
}

Background Lipedema is a condition consisting of painful bilateral increases in subcutaneous fat and interstitial fluid in the limbs with secondary lymphedema and fibrosis during later stages. Combined decongestive therapy (CDT) is the standard of care in most countries. Since the introduction of tumescent technique, liposuction has been used as a surgical treatment option. The aim of this study was to determine the outcome of liposuction used as treatment for lipedema.

Methods Twenty-five patients who received 72 liposuction procedures for the treatment of lipedema completed a standardized questionnaire. Lipedema-associated complaints and the need for CDT were assessed for the preoperative period and during 2 separate postoperative follow-ups using a visual analog scale and a composite CDT score. The mean follow-up times for the first postoperative follow-up and the second postoperative follow-up were 16 months and 37 months, respectively.

Results Patients showed significant reductions in spontaneous pain, sensitivity to pressure, feeling of tension, bruising, cosmetic impairment, and general impairment to quality of life from the preoperative period to the first postoperative follow-up, and these results remained consistent until the second postoperative follow-up. A comparison of the preoperative period to the last postoperative follow-up, after 4 patients without full preoperative CDT were excluded from the analysis, indicated that the need for CDT was reduced significantly. An analysis of the different stages of the disease also indicated that better and more sustainable results could be achieved if patients were treated in earlier stages.

Conclusions Liposuction is effective in the treatment of lipedema and leads to an improvement in quality of life and a decrease in the need for conservative therapy.

Keywords Lipedema; Lipoedema; Lipectomy
Correspondence: Mehran Dadras Department of Plastic Surgery, BG University Hospital Bergmannsheil, Ruhr-University Bochum, Bürkle de la Camp-Platz 1, 44789 Bochum, Germany

Tel: +49-234-3023560

Fax: +49-234-3026379

E-mail: mehran.dadras@rub.de

This article was presented the annual meeting of the German Society of Plastic, Reconstructive and Aesthetic Surgeons (DGPRÄC) that was held from October 1, 2015 to October 3, 2015 in Berlin, Germany.

No potential conflict of interest relevant to this article was reported.

\section{INTRODUCTION}

Lipedema was first described by Allen and Hines as a condition consisting of a pathological increase in subcutaneous fat and edema in the lower limb [1]. It almost exclusively affects women, and there are very few published case reports of men with li- 
pedema [2]. Lipedema shows familial clustering; one study proposed an autosomal dominant inheritance with sex limitation [3].

Clinically, lipedema can be characterized in most cases as a chronic disease that begins in puberty and takes a progressive course. Typically, there is a symmetric increase in subcutaneous fat in the upper and lower legs due to both hyperplasia and hypertrophy [4], which can be seen through magnetic resonance imaging or computed tomography $[5,6]$. The feet are spared, so there is an excess of fat at the ankle, also known as the "inverse shouldering effect." The upper limb is affected in up to $30 \%$ of cases, however it is rarely affected in isolation [7].

In addition to the increase in subcutaneous fat, there is an increase in capillary permeability and fragility, resulting in both extravasation of fluid and easy bruising [8]. Evidence has also been found of morphologic changes in the lymphatic system, such as microaneurysms; however, the pathophysiological significance remains unclear [9].

In contrast to primary lymphedema, the lymphatic system remains unimpaired in the initial stages of lipedema and can keep up with the increased amount of interstitial fluid [4]. Accordingly, lymphoscintigraphy has shown increased lymphatic transport in some patients [10]. During the later stages of lipedema, the amount of fluid produced exceeds the transport capacity of the lymphatic system, and the pressure of the fat tissue itself causes obstruction of the lymphatic vessels, resulting in secondary lymphedema $[10,11]$. Additionally, the deposition of protein-rich edema causes fibrosis of the tissue, further impairing lymphatic drainage. The term "lipolymphedema" is used to describe the combined pathology during these stages.

It is known that deposition of fat is positively correlated with stasis of blood and lymphatic fluid [12]. Therefore, a mutual interaction between adipose tissue and the lymphatic system exists.

Lipedema is diagnosed based on a clinical examination and anamnesis. Patients typically show disproportionality between a normal upper body and symmetrically enlarged lower limbs, and this is often accompanied by elevated body mass index (BMI) levels. Furthermore, the amount of fat in the affected areas is almost entirely unaffected by physical activity or dietary measures.

Typical clinical complaints include feeling of tension, pain upon pressure, and easy bruising. These symptoms are important in the differential diagnosis of lipedema, particularly in the common case where lipedema and obesity are present at the same time.

Lipedema can be classified into 3 clinical stages based on morphological appearance [13]: Stage I, a smooth skin surface with homogenous thickening of the subcutis; Stage II, a bumpy, wave-like skin surface with nodular structures in the thickened subcutis; Stage III, an increase in nodular changes and overhanging masses of tissue.

The condition is a major psychosocial burden for most patients, causing pain that often limits their capacity for exercise. In addition, standing for long periods of time and high temperatures are not tolerated well by those with lipedema, and in severe cases, the condition may cause absence from work or lead to occupational disability.

The most widely applied therapy for lipedema is combined decongestive therapy (CDT), which consists mainly of manual lymphatic drainage and wearing compression garments. It aims to reduce orthostatic edema and limit recurrence.

Classic dry liposuction cannot be applied to lipedema patients due to the potential injury to lymphatic vessels. However, the introduction of the tumescent technique in the 1980s has made the application of liposuction possible. Cadaver studies have shown markedly reduced injury to lymphatic structures when the tumescent technique was used [14].

In 1994, Rudkin and Miller [15] described liposuction in combination with skin and subcutaneous fat excisions as a treatment option for lipedema, in contrast to lymphedema. In 2002, liposuction alone was presented as a method to surgically reduce the pathological increase in subcutaneous fat [15-17].

The literature on lipedema, in general, is limited. Most articles are in German and fall under the disciplines of dermatology or phlebology. The present study aimed to examine the long-term results of liposuction in patients with lipedema who were treated in our plastic surgery clinic.

\section{METHODS}

From July 2010 to July 2013, 33 female patients received a total of 104 liposuction procedures in our clinic as treatment for lipedema. Patients were classified preoperatively into the 3 clinical stages described above.

At the end of 2013, these patients received a standardized questionnaire composed of 18 items. Visual analog scales (VAS) from 0 to 10 in increments of 0.5 were used to assess the severity of spontaneous pain, pain upon pressure, feeling of tension, bruising, cosmetic impairment, and general impairment of quality of life before and after liposuction treatment. Questions were also asked regarding weight, the frequency of manual lymphatic drainage per month, and the number of hours per day the patient wore compression garments. These last two values were added together to give a CDT score. In 2015, the assessment was repeated using the same questions but assessed only the 
current state of the patients' symptoms.

Twenty-five patients responded in 2013 and were included in this study, and all 25 patients were available for follow-up in 2015.

The first postoperative follow-up was performed between 4 and 34 months after patients received their last liposuction procedure, with a mean follow-up time of 16 months. The second postoperative follow-up was performed between 25 and 56 months after the last liposuction procedure, with a mean followup time of 37 months.

For each patient, lipedema diagnosis had been clinically confirmed by a lymphologist, ruling out other lymphatic diseases. Each procedure was covered by health insurance after a medical proposal was accepted, and the patient had typically already received at least 6 months of CDT without improvement of symptoms.

Tumescent liposuction was performed using saline with epinephrine $(1: 1,000,000)$ after obtaining informed consent. All liposuctions were performed as inpatient procedures, and general anesthesia was used during the multi-hour procedures to increase patient comfort and reduce operation time. Antibiotics were administered as a single shot for perioperative prophylaxis only.

Patients were told to bring their compression garments, which were put on immediately after liposuction. New garments were measured 3 weeks after liposuction and after swelling had decreased, and manual lymphatic drainage was allowed after postoperative day 2.

Statistical analyses of complaint severity and the CDT score were performed in SPSS ver. 21.0 for Mac (IBM Corp., Armonk, NY, USA) using repeated-measures analysis of variance (ANOVA) with the Bonferroni correction after meeting the criteria of the Mauchly test of sphericity. All the tests were 2-sided, with $\alpha=0.05$ and $\mathrm{P}<0.05$ considered statistically significant.

\section{RESULTS}

The age at the first performed liposuction ranged from 23 to 64 years, with a median of 45 years. BMI upon presentation ranged from 24.5 to $50.6 \mathrm{~kg} / \mathrm{m}^{2}$, with a mean of $35.3 \mathrm{~kg} / \mathrm{m}^{2}$. After treatment, BMI ranged from 22.7 to $47.2 \mathrm{~kg} / \mathrm{m}^{2}$, with a mean of 33.9 $\mathrm{kg} / \mathrm{m}^{2}$.

All patients had lipedema of the lower limb. Additional upper limb involvement was present in 9 patients (36\%). One patient

Table 1. Results from the analysis of the questionnaires $(n=25)$

\begin{tabular}{|c|c|c|c|c|c|c|}
\hline \multirow{2}{*}{ Measured variable } & \multicolumn{2}{|c|}{ Preoperative } & \multicolumn{2}{|c|}{ Postoperative 1} & \multicolumn{2}{|c|}{ Postoperative 2} \\
\hline & Mean & SD & Mean & SD & Mean & SD \\
\hline \multicolumn{7}{|l|}{ Complaintsa) } \\
\hline Spontaneous pain & 7.20 & 1.46 & 3.70 & 1.79 & 4.28 & 2.10 \\
\hline Stage $\|(n=11)$ & 7.00 & 1.40 & 3.36 & 2.01 & 4.00 & 2.18 \\
\hline Stage III $(n=13)$ & 7.54 & 1.44 & 4.12 & 1.58 & 4.61 & 2.13 \\
\hline Sensitivity to pressure & 7.38 & 1.79 & 3.98 & 1.83 & 4.42 & 2.08 \\
\hline Stage $\|(n=11)$ & 7.05 & 1.29 & 3.50 & 1.97 & 3.82 & 2.27 \\
\hline Stage III ( $n=13)$ & 7.77 & 1.56 & 4.46 & 1.71 & 4.81 & 1.90 \\
\hline Feeling of tension & 7.52 & 1.36 & 3.26 & 2.28 & 4.06 & 2.18 \\
\hline Stage $\|(n=11)$ & 7.09 & 1.00 & 3.14 & 2.47 & 4.00 & 2.35 \\
\hline Stage III (n=13) & 8.08 & 1.35 & 3.62 & 2.07 & 4.35 & 2.01 \\
\hline Bruising & 6.96 & 1.58 & 4.36 & 1.91 & 4.64 & 1.83 \\
\hline Stage $\|(n=11)$ & 6.82 & 1.54 & 3.86 & 1.78 & 4.46 & 1.89 \\
\hline Stage III $(n=13)$ & 7.15 & 1.70 & 4.65 & 2.03 & 4.66 & 1.85 \\
\hline Cosmetic impairment & 8.98 & 0.81 & 5.10 & 1.93 & 7.36 & 1.66 \\
\hline Stage $\|(n=11)$ & 8.68 & 0.72 & 4.55 & 1.97 & 6.96 & 1.52 \\
\hline Stage III $(n=13)$ & 9.31 & 0.78 & 5.58 & 1.91 & 7.85 & 1.69 \\
\hline Impairment to quality of life & 8.38 & 1.06 & 4.30 & 1.80 & 5.16 & 1.60 \\
\hline Stage $\|(n=11)$ & 8.22 & 1.35 & 4.09 & 2.12 & 4.64 & 1.89 \\
\hline Stage III $(n=13)$ & 8.62 & 0.71 & 4.42 & 1.63 & 5.46 & 1.23 \\
\hline CDT score ${ }^{b)}$ & 20.48 & 4.13 & 16.38 & 6.97 & 13.90 & 7.32 \\
\hline Stage $\|(n=9)$ & 21.22 & 4.58 & 13.33 & 9.15 & 12.00 & 9.89 \\
\hline Stage III $(n=11)$ & 19.91 & 4.06 & 18.55 & 3.93 & 14.90 & 4.57 \\
\hline
\end{tabular}

$\mathrm{SD}$, standard deviation; CDT, combined decongestive therapy.

${ }^{a}$ Visual analog scale of symptom severity ranging from 0 to 10 in increments of 0.5 , with 10 being the most severe; ${ }^{b}$ The CDT score was calculated as the sum of the number of manual lymphatic drainage sessions per month and the number of hours spent wearing compression garments per day. Four patients were excluded who did not receive full CDT preoperatively. 
had stage I lipedema, 11 patients had stage II lipedema, and 13 patients had stage III lipedema.

On average, patients received 3 procedures, with a range of 1 to 7 procedures. A total of 72 liposuctions were performed on the 25 patients. In 41 liposuctions, a vibration-assisted device was used, and in 31 liposuctions, a water jet-assisted device was used.

The operation time, which included infiltration, was $116 \mathrm{~min}$ utes on average and ranged from 58 to 251 minutes.

The mean volume of removed fat per liposuction was 3,106 $\mathrm{mL}$ (range, 1,450-6,600 mL) and the mean volume of total removed fat per patient was $9,914 \mathrm{~mL}$ (range, 4,000-19,850 mL).

One patient developed erysipelas after liposuction, which required antibiotic treatment. However, there were no other complications during the study period. Therefore, the complication rate was $1.39 \%$.

\section{Complaints}

Table 1 shows that patients reported substantial lipedema-associated complaints preoperatively. Spontaneous pain was reported with a mean VAS score of 7.2 (standard deviation [SD], 1.46); the equivalent of "severe" to "very severe" spontaneous pain. Sensitivity to pressure and feeling of tension were reported with mean VAS scores of 7.38 (SD, 1.79) and 7.52 (SD, 1.36), respectively, falling within the "very severe" range. The reported cosmetic impairment ranged from "severe" to "unbearable," resulting in a mean VAS score of 8.98 (SD, 0.81). General impairment to quality of life was also reported as "very severe," with a mean VAS score of 8.38 (SD, 1.06).

Fig. 1 shows that the severity of all analyzed complaints was significantly reduced over the course of liposuction treatment by the time of the first postoperative follow-up. All but 1 of the patients reported a reduction in spontaneous pain (the chief complaint in lipedema), with a mean difference in VAS score of 3.5 (95\% confidence interval [CI], 2.83-4.17). Furthermore, all but 1 of the patients reported a reduction in impairment of quality of life, with a mean difference in VAS score of 4.08 (95\% CI, 3.12-5.04).

The Bonferroni-corrected P-value was $<0.001$ for all 6 complaints. At the second postoperative follow-up, only the severity of cosmetic impairment significantly increased since the first postoperative follow-up, and there was significant improvement in all symptoms between the preoperative period and the second postoperative follow-up. Fig. 2 shows a comparative subgroup analysis of general impairment to quality of life for patients with stage II lipedema and stage III lipedema. This symptom was chosen for analysis because it was the most important complaint. While a significant reduction in the severity of the complaint from the preoperative period to the first postoperative follow-up was observed for both stage II and stage III patients, only stage III patients experienced a significant increase in the severity of the complaint from the first postoperative follow-up to the second postoperative follow-up.

\section{Conservative therapy}

Three patients did not wear compression garments preoperatively and only started wearing them after liposuction in order to retain the results of the treatment. One patient who did not receive manual lymphatic drainage preoperatively received it postoperatively. Twenty-one patients regularly received manual lymphatic drainage and wore compression garments preoperatively. For these patients, CDT scores were calculated as the sum of manual lymphatic drainage sessions per month and hours spent wearing compression garments per day.

At the second postoperative follow-up, the CDT scores of 14 patients had decreased after liposuction treatment, with 3 patients no longer in need of further conservative therapy. Three patients showed no change in their CDT scores, while 4 patients showed an increase in their CDT scores.

For all patients who received full CDT preoperatively, the mean CDT score had decreased from 20.48 (SD, 4.13) during the preoperative period to $16.38(\mathrm{SD}, 6.97)$ during the first postoperative follow-up, and then decreased further to 13.9 (SD, 7.32) during the second postoperative follow-up. Fig. 2 shows that in the repeated-measures ANOVA, only the reduction in CDT score from the preoperative period to the second postoperative follow-up was found to be significant $(\mathrm{P}=0.011)$.

A comparative subgroup analysis of patients with stage II lipedema and stage III lipedema showed a significant decrease in the CDT score from the preoperative period to the second postoperative follow-up for stage II patients only. The reduction in $\mathrm{CDT}$ score from the preoperative period to the second postoperative follow-up for stage III patients was barely non-significant $(\mathrm{P}=0.051)$.

\section{DISCUSSION}

In most countries, $\mathrm{CDT}$ is the first line of therapy for lipedema. Though this treatment aims to stop the progression of the disease and reduce edema, many patients still see an increase in subcutaneous fat and aggravation of symptoms.

When the tumescent technique for liposuction was introduced in the late 1980s [18], the safety of liposuction improved and injury to lymphatic vessels was reduced $[14,19]$. Subsequently, liposuction became an option in treating lipedema and reducing the amount of fat tissue. 


\section{Fig. 1. Complaints}

Box plots of the complaints before and after liposuction with accompanying P-values $(n=25)$. VAS, visual analog scales.
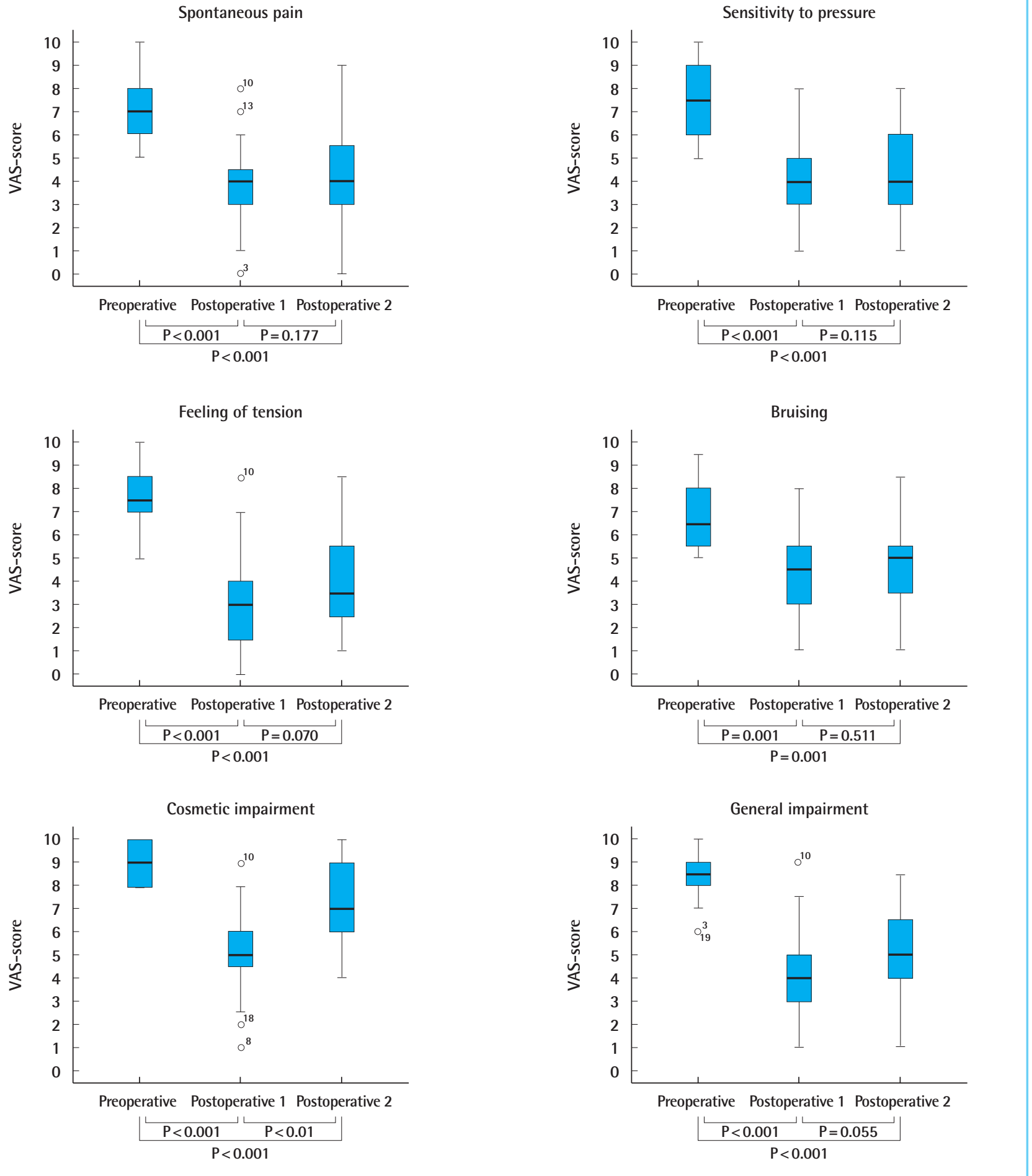

Our study showed that liposuction was an effective treatment for lipedema with good long-term outcomes. Our findings support the results of previous studies, such as Schmeller et al. [20], Rapprich et al. [21], and the recent long-term study by Baumgartner et al. [22].
However, unlike the aforementioned studies, the data for the preoperative period were collected retrospectively, representing a possible bias.

An average operation time for liposuction under tumescent anesthesia that includes infiltration has been reported as 5.5 


\section{Fig. 2. Combined decongestive therapy scores and subgroup analyses}

Box plots of the combined decongestive therapy (CDT) scores before and after liposuction ( $n=21,4$ patients excluded who did not receive full CDT preoperatively) and subgroup analyses of the visual analog scale (VAS) scores for general impairment of quality to life by stage of lipedema ( $n=11$ for stage II lipedema, $n=13$ for stage III lipedema) and the CDT scores by stage of lipedema ( $n=9$ for stage II lipedema, $n=11$ for stage III lipedema).

General impairment, Stage II subgroup $(n=11)$
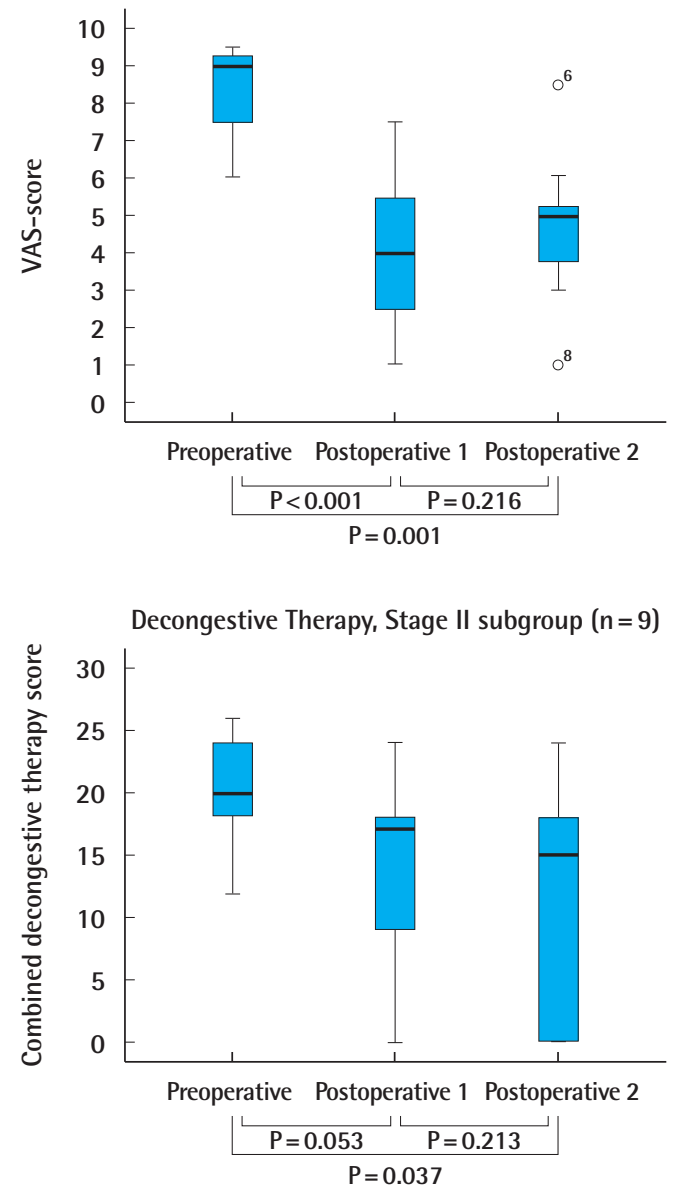

hours. In our study, the average operation time under general anesthesia that included infiltration was roughly 2 hours, yielding comparable amounts of removed fat [22].

In accordance with the Practice Advisory on Liposuction published by the American Society of Plastic Surgeons, we believe that general anesthesia has advantages over tumescent anesthe-
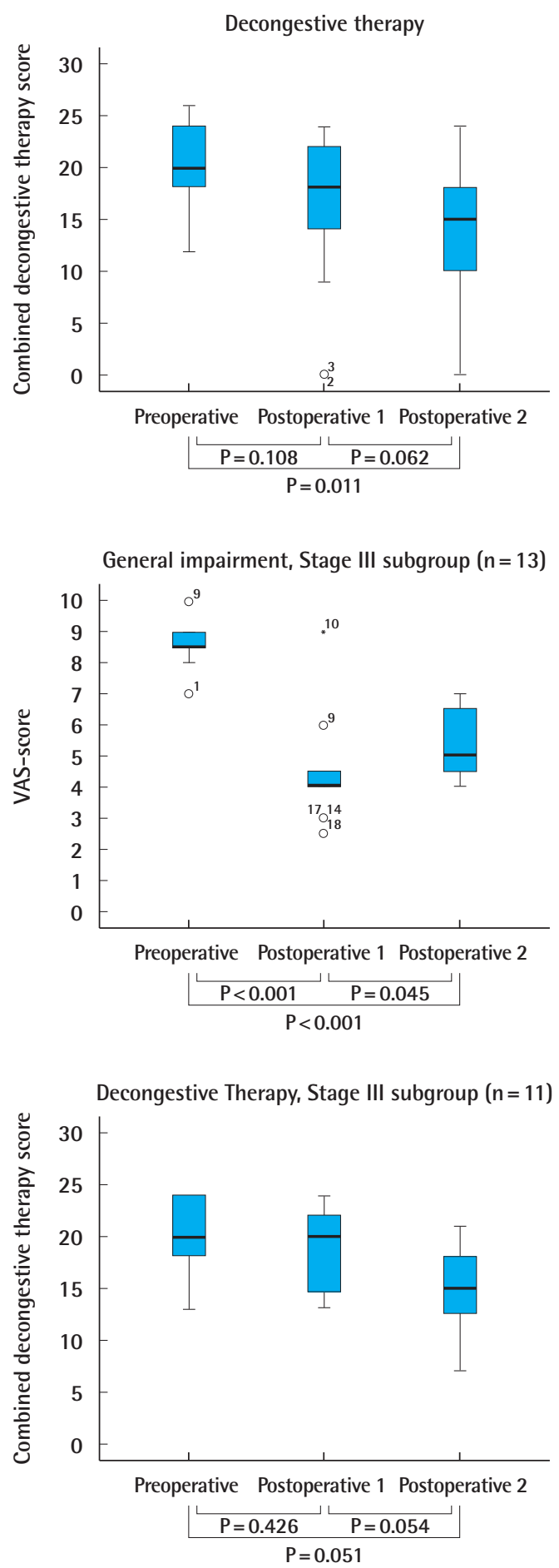

sia for long and complex liposuctions, such as those used to treat lipedema [23].

General anesthesia has been shown to be a safe procedure and has a decreased risk of aspiration and airway complications when compared to sedation [24]. This may be needed when operating under tumescent anesthesia to alleviate patient dis- 


\section{Fig. 3. Case example 1}

A 24-year-old patient with stage II lipedema preoperatively (A) and 10 months after 2 liposuctions (B). A total of 8,800 mL of fatty tissue was removed from her legs.
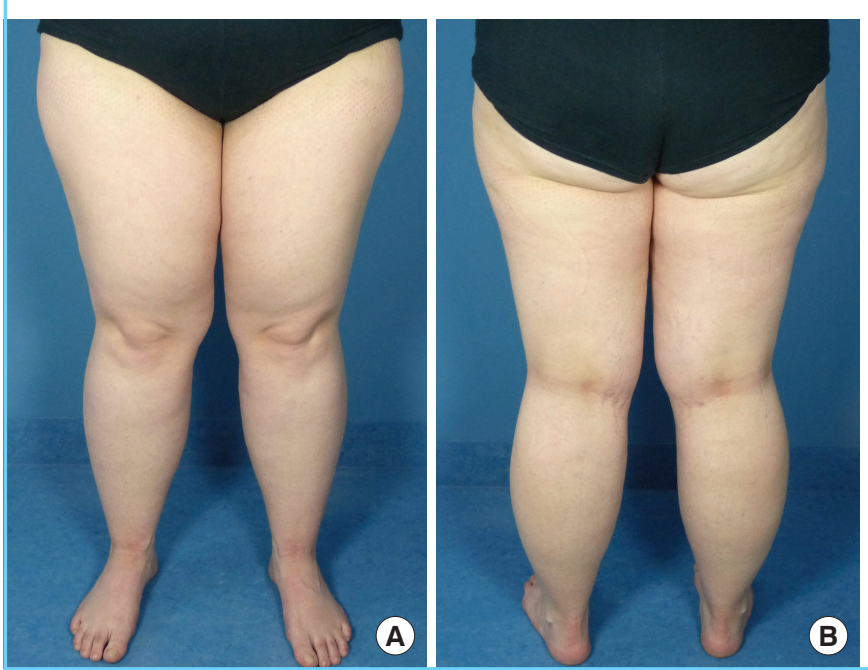

\section{Fig. 4. Case example 2}

A 65-year-old patient with stage III lipedema preoperatively (A) and 6 months after 3 liposuctions (B). A total of 11,600 mL of fatty tissue was removed from her legs.
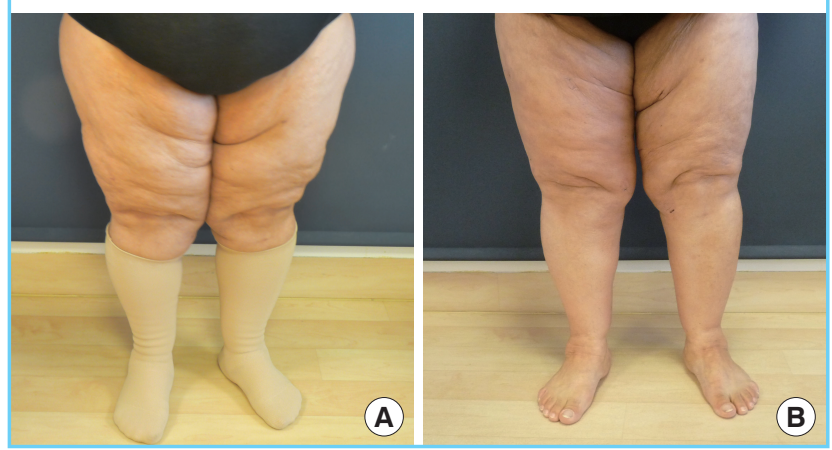

comfort.

During our study, there was only 1 minor infectious complication from the 72 liposuctions, resulting in a complication rate of $1.4 \%$. Schmeller et al. [20] saw the same rate of infection in 349 liposuctions used to treat lipedema, and Rapprich et al. [21] reported just 1 case of deep vein thrombosis during 15 years of surgical treatment for lipedema. In general, liposuction using the tumescent technique carries a low surgical risk and has been shown to be a safe and appropriate procedure [25].

The patient examples in Figs. 3 and 4 show that liposuction led to a marked reduction in the volume of fatty tissue in the affected limbs and the disproportionality of these limbs to the rest of the body. Although this was not measured quantitatively in our study, Rapprich et al. [21] were able to show volume reduction in lipedema patients treated with liposuction using 3-dimensional volumetry.
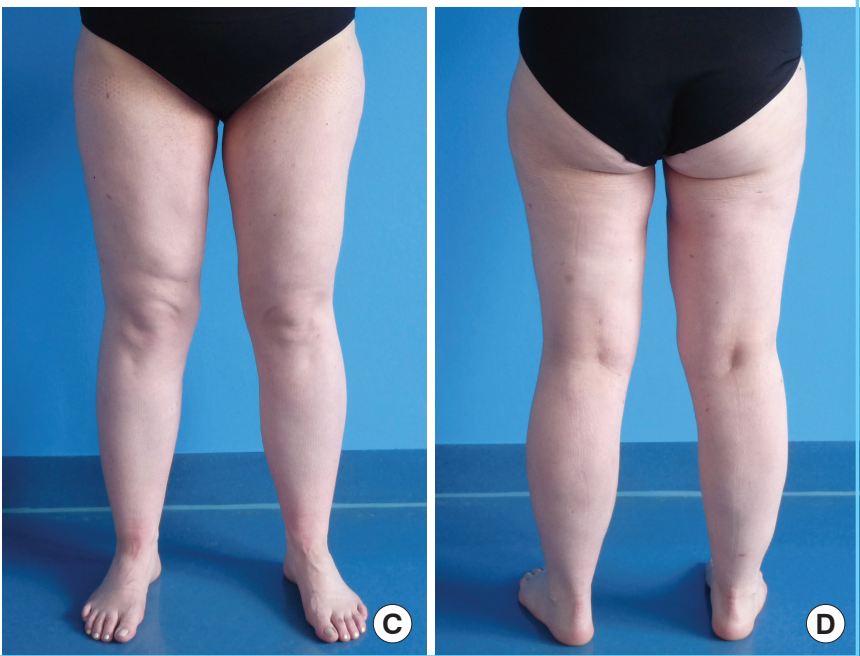

The severity of all major symptoms of lipedema was significantly reduced, and there was a significant reduction in the severity of impairment of quality to life, as reported by the patients.

This corresponds with our clinical experiences, where we have found that lipedema patients benefit greatly from liposuction treatment and display a very high level of gratitude.

After extensive liposuction, the resulting excess skin tissue may harm the long-term aesthetic outcome. This may be a possible explanation for the increase in the VAS score for cosmetic impairment that was measured during the second postoperative follow-up and was perceived to be caused by the disease. Lifting operations should be considered for these patients, taking all necessary precautions to preserve lymphatic vessels.

We propose a simple score to quantify the need for CDT. From our clinical experience and that of our cooperating lymphologists, we have found that lipedema patients wear compression garments for roughly 8 to 10 hours per day on average, and patients will typically receive 2 manual lymphatic drainage sessions per week. Deviations from these patterns depend on symptom severity and distribution. Thus, summing the number of hours spent wearing compression garments per day and the number of manual lymphatic drainage sessions per month is an easy way to quantify $\mathrm{CDT}$ in a single score. Using this score, we were able to show for the first time that liposuction for patients with lipedema led to a significant decrease in the need for conservative lipedema treatment and also improved the quality of life for these patients. Previous studies that had already shown that liposuction may result in a decreased need for conservative lipedema treatment did not quantify this need or test for signifi- 
cance $[20,21]$.

Our data suggest that liposuction treatment for stage II lipedema provides a more sustainable reduction in the impairment of quality to life and a larger decrease in the need for conservative therapy than liposuction treatment for stage III lipedema.

Due to the development of secondary lymphedema and the irreversible damage to the lymphatic system that occurs in later stages of the disease, liposuction should be implemented as part of the standard therapy for lipedema at early stages. This will prevent disease progression, improve quality of life, and reduce the need for decongestive therapy.

\section{ORCID}

Mehran Dadras https://orcid.org/0000-0003-1283-2982

\section{REFERENCES}

1. Allen EV, Hines EA. Lipedema of the legs: a syndrome characterized by fat legs and orthostatic edema. Proc Staff Meet Mayo Clin 1940;15:184-7.

2. Chen SG, Hsu SD, Chen TM, et al. Painful fat syndrome in a male patient. Br J Plast Surg 2004;57:282-6.

3. Child AH, Gordon KD, Sharpe P, et al. Lipedema: an inherited condition. Am J Med Genet A 2010;152:970-6.

4. van Geest AJ, Esten SC, Cambier JP, et al. Lymphatic disturbances in lipoedema. Phlebologie 2003;32:138-42.

5. Dimakakos PB, Stefanopoulos T, Antoniades P, et al. MRI and ultrasonographic findings in the investigation of lymphedema and lipedema. Int Surg 1997;82:411-6.

6. Vaughan BF. CT of swollen legs. Clin Radiol 1990;41:2430.

7. Herpertz U. Lipedema. Z Lymphol 1995; 19:1-11.

8. Curri SB, Merlen JF. Microvascular disorders of adipose tissue. J Mal Vasc 1986;11:303-9.

9. Amann-Vesti BR, Franzeck UK, Bollinger A. Microlymphatic aneurysms in patients with lipedema. Lymphology 2001;34:170-5.

10. Tiedjen KU, Schultz-Ehrenburg U. Isotopenlymphographische befunde beim lipodem. In: Holzmann H, editor. Dermatologie und nuklearmedizin. Berlin: Springer-Verlag; 1985. p.432-8.

11. Lohrmann C, Foeldi E, Langer M. MR imaging of the lym- phatic system in patients with lipedema and lipo-lymphedema. Microvasc Res 2009;77:335-9.

12. Ryan TJ. Lymphatics and adipose tissue. Clin Dermatol 1995; 13:493-8.

13. Meier-Vollrath I, Schmeller W. Lipoedema: current status, new perspectives. J Dtsch Dermatol Ges 2004;2:181-6.

14. Hoffmann JN, Fertmann JP, Baumeister RG, et al. Tumescent and dry liposuction of lower extremities: differences in lymph vessel injury. Plast Reconstr Surg 2004;113:718-24.

15. Rudkin GH, Miller TA. Lipedema: a clinical entity distinct from lymphedema. Plast Reconstr Surg 1994;94:841-7.

16. Sattler G. Liposuction in lipoedema. Ann Dermatol Venerol 2002;129:1S103.

17. Rapprich S, Loehnert M, Hagedorn M. Therapy of lipoedema syndrome by liposuction under tumescent local anaesthesia. Ann Dermatol Venerol 2002;129:1S71.

18. Klein JA. The tumescent technique for liposuction surgery. Am J Cosmetic Surg 1987;4:1124-32.

19. Stutz JJ, Krahl D. Water jet-assisted liposuction for patients with lipoedema: histologic and immunohistologic analysis of the aspirates of 30 lipoedema patients. Aesthetic Plast Surg 2009;33:153-62.

20. Schmeller W, Hueppe M, Meier-Vollrath I. Tumescent liposuction in lipoedema yields good long-term results. $\mathrm{Br} \mathrm{J}$ Dermatol 2012;166:161-8.

21. Rapprich S, Dingler A, Podda M. Liposuction is an effective treatment for lipedema-results of a study with 25 patients. J Dtsch Dermatol Ges 2011;9:33-40.

22. Baumgartner A, Hueppe M, Schmeller W. Long-term benefit of liposuction in patients with lipoedema: a follow-up study after an average of 4 and 8 years. Br J Dermatol 2016; 174:1061-7.

23. Iverson RE, Lynch DJ, American Society of Plastic Surgeons Committee on Patient S. Practice advisory on liposuction. Plast Reconstr Surg 2004;113:1478-90.

24. Hoefflin SM, Bornstein JB, Gordon M. General anesthesia in an office-based plastic surgical facility: a report on more than 23,000 consecutive office-based procedures under general anesthesia with no significant anesthetic complications. Plast Reconstr Surg 2001;107:243-51.

25. Habbema L. Safety of liposuction using exclusively tumescent local anesthesia in 3,240 consecutive cases. Dermatol Surg 2009;35:1728-35. 